\title{
Dialisi peritoneale: ecco la dialisi che vorrei fare!
}

\author{
Gianpaolo Amici
}

S.O.C. Nefrologia e Dialisi, Ospedale di San Daniele, ASS4 "Medio Friuli”, Udine

\begin{abstract}
Peritoneal dialysis: here is the dialysis I WOUld like!
Abstract. Unfortunately, when a patient needs to start dialysis and choose its modality hemodialysis appears to be a solid and well organized method, with several additional services all well appreciated by both patients and families. By contrast, peritoneal dialysis seldom reaches a similar level of organization and structural investments. To offer patients and their families a valid alternative we need to go some steps further in the field of home services, particularly those dedicated to disabilities and chronic conditions.
\end{abstract}

Key words: Peritoneal dialysis, Home services, Chronicity, Disability

Conflict of interest: None.

Financial support: None.

Accettato: 16 Agosto 2013

"Ma perché devo fare dialisi a casa? Io voglio fare emodialisi in ospedale, con le infermiere che si occupano di tutto e con i pulmini che mi vengono a prendere e mi riportano a casa. Poi posso riposare a letto e guardare la televisione per tutto il tempo. Per non parlare del fatto che ho quattro giorni liberi che posso utilizzare come voglio; inoltre, le analisi del sangue le fanno direttamente in dialisi, mi fanno trovare il pacchetto dei farmaci senza che io debba andare dal mio medico di base, mi servono tè o caffè e mi danno anche uno spuntino, se voglio. Per i miei familiari non sono un peso, loro non devono fare nulla, e, se ho un problema, posso parlare con un medico e, all'occorrenza, essere direttamente ricoverato, perché sono in ospedale, sono al sicuro".

Sono io stesso che scrivo queste righe, mettendomi nei panni di un paziente, ma qualsiasi nefrologo le sa queste cose, le conosce bene, le vive e le organizza, se ne occupa. Ogni nefrologo conosce per prima l'emodialisi e questa è, per la maggioranza di noi, il principale campo di lavoro e di esperienza.

Dal momento che lo sviluppo delle competenze professionali avviene nel corso degli anni di lavoro post-specializzazione, si comprende come la conoscenza della dialisi peritoneale sia, in molti casi, solo parziale o solo teorica.

Allora, quali possono essere le chiavi dello sviluppo, della pratica e della conoscenza della dialisi peritoneale a questo punto non solo in Italia ma in tutta Europa?
Il problema, per come lo osservo da nefrologo appassionato di dialisi peritoneale da sempre, ha molte facce e proverò ad approfondirle suggerendo possibili soluzioni.

Per prima cosa, l'organizzazione della dialisi peritoneale dovrebbe ricevere la stessa dignità, la stessa attenzione e gli stessi investimenti di ogni servizio di emodialisi. Sembra una frase scontata, ma tutti sanno quanto difficile sia avere infermieri, locali, magazzini, linee telefoniche dedicate e attività di segreteria. Tutti sanno che questi servizi, se presenti, devono sempre essere condivisi con ambulatori speciali di predialisi trapianto, DH e libera professione. Devono essere condivisi gli spazi ambulatoriali e il personale dedicato, bisogna accettare personale in part-time, bisogna, insomma, cambiare divisa ed esercito secondo le giornate.

$\mathrm{E}$ il tessuto sociale? Le famiglie sono più piccole e gli anziani sono spesso in coppia, ma entrambi affetti da pluripatologie e invalidità. Allora la risposta è data dai servizi; quali sono, in pratica, i servizi offerti alla dialisi peritoneale rispetto ai servizi per l'emodialisi? Ci sono visite domiciliari, assistenza infermieristica, assistenza sociale per le esigenze della vita di tutti i giorni, cooperative di infermieri per la dialisi peritoneale assistita a domicilio, posti riservati nelle case di riposo e aree pubbliche dove poter fare dialisi peritoneale in sicurezza senza restare a casa?

E per i prelievi ematochimici e le ricette si deve andare dal medico di base e fare la fila come tutti gli altri? E, quando si va in ospedale, ci sono i trasporti o ci sono i posti macchina 
per invalidi sufficienti e vicini per i pazienti in dialisi peritoneale?

Ecco, allora, le risposte per non perdere questa partita che ormai è in gioco da tre decenni. Facili risposte; sì, vanno bene la dedizione, lo studio e la conoscenza pratica, ma servono davvero i servizi per affrontare la disabilità e la cronicità e un supporto per le persone sole e per le famiglie che sia modulabile secondo le esigenze con volontariato sociale, assistenza sociale per le pulizie, la spesa e il bucato, visite domiciliari infermieristiche per individuare i bisogni sanitari, assistenza alla dialisi sporadica o continuativa e, perché no, assistenza a pagamento, se richiesta. Possono essere, infine, utili case della dialisi per fare la dialisi peritoneale in spazi organizzati, case di riposo con posti permanenti dedicati e anche posti temporanei o solo notturni. Voglio, infine, ricordare che disabilità e assistenza non devono essere solo associati agli anziani ma che spesso i nefropatici giovani presentano grandi bisogni.

Come si può capire da queste mie poche righe, la dialisi peritoneale ha bisogno di sensibilità sociale, ha bisogno di amministrazioni disponibili a creare e organizzare nuovi servizi, ha bisogno di nefrologi proiettati nel territorio e non arroccati nei reparti in ospedale. Come osservazione finale, posso solo notare che questi percorsi non dovrebbero essere inventati da ognuno di noi ma, sia nelle creazioni che nelle difficoltà, dovrebbero essere riconosciuti come patrimonio comune della nefrologia.

"Ma perché devo fare l'emodialisi in ospedale... io sto comodo a casa mia, non devo aspettare il pulmino per andare in ospedale, non devo aspettare l'orario di attacco e di stacco, non devo aspettare il pulmino per il ritorno. Accidenti, non sempre le punture della fistola vanno bene e non posso certo scegliere l'infermiere che mi attacca. Invece, a casa mia, decido io quando iniziare la dialisi e con le persone che mi seguono costantemente vado molto d'accordo. Vengono a casa mia a fare i prelievi, mi portano spesso i farmaci e mi fanno la spesa e le pulizie; so che, quando i miei familiari non possono, ci sono persone che mi aiutano e so che, una volta al mese, il medico della dialisi viene a visitarmi. Certo, la dialisi non è una bella esperienza e non è una bella malattia, la vita è dura in dialisi, ma farla a casa in tutta comodità me la rende più accettabile o, almeno, un po'di più'”.

Ecco quello che vorrei sentire, ecco quello che vorrei fare con il mio lavoro!

\section{Riassunto}

Quando il paziente è costretto a fare dialisi e a scegliere la metodica, l'emodialisi si presenta solida e ben organizzata, con numerosi servizi accessori che vengono apprezzati dalle famiglie e dai pazienti. La dialisi peritoneale raramente raggiunge lo stesso livello di organizzazione e di investimenti strutturali. Per dare ai pazienti e alle famiglie una buona alternativa, devono essere fatti passi avanti come organizzazione territoriale e servizi, affrontando con serietà le necessità della cronicità e della disabilità.

Parole chiave: Dialisi peritoneale, Organizzazione territoriale, Cronicità, Disabilità

Dichiarazione di conflitto di interessi: L'Autore dichiara di non avere conflitto di interessi.

Contributi economici degli autori: L'Autore dichiara di non aver ricevuto sponsorizzazioni economiche per la preparazione dell'articolo.

Indirizzo degli Autori:

Dr. Gianpaolo Amici

Unità di Nefrologia e Dialisi

Ospedale di San Daniele

ASS4 Medio Friuli

33100 Udine

gianpaolo.amici@mediofriuli.it 